\title{
EL TRABAJO EN EQUIPO Y LA FORMACIÓN DEL GEÓGRAFO. PROBLEMAS Y RETOS*
}

\author{
Ana Isabel Escalona Orcao y Blanca Loscertales Palomar \\ Dpto. de Geografía y Ordenación del Territorio. Universidad de Zaragoza
}

\begin{abstract}
Resumen: La implantación de los nuevos títulos de grado es una de las consecuencias principales de la entrada en vigor del Espacio Europeo de Educación Superior. En este nuevo marco, el aprendizaje de diferentes competencias específicas y genéricas va a ser un referente común para la formación de los estudiantes de las diferentes titulaciones y sistemas universitarios europeos. Esta publicación se centra en la titulación de Geografía y en la competencia trabajo en equipo, modo en el que se desarrolla habitualmente el quehacer profesional del geógrafo. Después de realizar unas consideraciones iniciales sobre el potencial formativo de dicha competencia, se expone una metodología para su incorporación a la enseñanza universitaria que contempla el diseño, realización de actividades y evaluación del aprendizaje en las diferentes asignaturas. El artículo termina con una reflexión sobre esa renovación que se vislumbra ya a muy corto plazo en el Espacio Europeo de Educación Superior.
\end{abstract}

Palabras clave: Espacio Europeo de Educación Superior, Competencias genéricas, Metodología docente, Trabajo en equipo.

\begin{abstract}
An important step in the process of designing new degrees for the European Higuer Education Area is the identification of learning outcomes and competences and its translation into the curricula. This paper approaches teaching and learning of the generic competence teamwork in the framework of the new Spanish degree in Geography. After discussing a rationale for the development of teamwork skills within the Geography degree, the article provides a methodological model and teaching materials including statement of learning outcomes, developing of assesement methods and organisation of academic activities. The article finishes emphasising skills learning and inviting Geography teachers to undertake this task in their University courses.
\end{abstract}

Key words: European Higuer Education Area, Generic skills Teaching methodology, teamwork.

* Recibido: 27-1-2006. Aceptado: 8-1-2007. 


\section{Introducción}

Uno de los aspectos de la entrada en vigor del Espacio Europeo de Educación Superior, es la implantación de los nuevos títulos de grado. En el caso del título de Geografía, como en los restantes, supone significativos cambios respecto de los títulos hasta ahora vigentes. Su diseño se enmarca en la determinación previa de las necesidades sociales que tiene que satisfacer la titulación, estableciendo cuáles son los rasgos que deben caracterizar a sus egresados. Los contenidos habrán de describir de modo preciso qué aporta el aprendizaje y se encaminan a que los titulados encajen en los diferentes perfiles profesionales. Los resultados de los aprendizajes en cada una de las titulaciones se expresan en forma de competencias, tanto específicas -propias de un determinado campo de estudio- como genéricas -referidas a cualidades diversas y socialmente cada vez más apreciadas -(VV.AA, 2003).

La formación en competencias genéricas ha estado presente desde siempre en el trabajo de los profesores de Geografía. Lo que se nos pide ahora es que las enseñemos de forma explícita y sistemática (Gravestock y Healey, 2000). De este modo los estudiantes serán conscientes de que van a ser formados en aspectos personal y profesionalmente valiosos junto con los contenidos propios de la disciplina. Todo ello contribuirá a mejorar la preparación y la futura inserción profesional de los egresados. El trabajo en equipo, objeto de este artículo, es una competencia muy necesaria para el geógrafo, cuya actividad profesional se desarrolla habitualmente en un marco interdisciplinar y de estrecha colaboración con otros especialistas. Además el aprendizaje de esta competencia mejora otras muchas capacidades, habilidades y destrezas que se consideran también muy importantes en la formación integral de un universitario. Es el caso de la capacidad de liderazgo, de escucha activa, de discusión, de crítica, de comunicación, de responsabilidad, de organización del tiempo, de cooperación y de relación social.

El artículo ofrece a los profesores universitarios de Geografía un ejemplo aplicable de enseñanza y aprendizaje de la competencia trabajo en equipo en sus asignaturas. Se sigue la pauta iniciada en otra publicación anterior de Geographicalia (v. Escalona y Loscertales, 2005b) en la que se planteaba un marco general para diseñar la enseñanza de cualquier competencia genérica. Las actuaciones que se proponen en este artículo responden a las recomendaciones recogidas en el documento Propuestas para la renovación de las metodologías educativas en la Universidad (CRUE, 2006). El artículo comienza con unas consideraciones sobre la competencia trabajo en equipo y su importancia en el quehacer profesional del geógrafo. A continuación se propone una metodología para el diseño del aprendizaje y se dan pautas para su incorporación de forma sistemática y con facilidad en las diferentes asignaturas de nuestra titulación. 


\section{La competencia trabajo en equipo y su interés en el quehacer profesional y en la formación del geógrafo}

El trabajo en equipo -capacidad para integrarse con otros en el logro de unos objetivos comunes, distribuyendo y compartiendo responsabilidades, conforme a habilidades que conviene sean complementarias (VV.AA, 2001: 28 y ss.) - es una competencia interpersonal, que favorece los procesos de interacción social y comunicación. Aprender a trabajar con otros es una oportunidad para potenciar otras habilidades y capacidades como las de planificación del tiempo y de los recursos, cumplimiento de responsabilidades y tareas, escucha activa, negociación, liderazgo, comunicación y autoevaluación entre otras (Bradford et al., 2000).

Diferentes encuestas realizadas en el marco de la adaptación al Espacio Europeo de Educación Superior dejan clara la alta valoración de la competencia trabajo en equipo. Así quedó claramente patente en la incluida en el informe Tuning, (VV.AA: 2003: 105). En la encuesta incorporada al proyecto de título de grado en Geografía y Ordenación del Territorio se obtuvieron resultados semejantes. La competencia trabajo en equipo recibió una valoración global de 3’2 sobre 4 (Agencia..., op. cit: 192), reflejando que se trata de una competencia necesaria para desarrollar las tareas propias de los geógrafos, centradas en cuestiones que son contempladas también por especialistas de otras disciplinas. Así sucede con el desarrollo territorial y planificación, medio ambiente, demografía y estudios sociales, desarrollo regional, tecnologías de la información geográfica entre otros campos de actividad. Por ello las expectativas profesionales de los geógrafos en estos campos dependen de una formación de calidad y de la capacidad para trabajar en equipos interdisciplinares aportando la especificidad de la disciplina (Tarroja, 2005).

La opinión de los estudiantes va en la misma línea. Según los resultados de la encuesta realizada en el Departamento de Geografía de la Universidad de Zaragoza, por las autoras, el trabajo en equipo fue puntuado con 4 y 5 (en una escala de 1 a 5) por el $70 \%$ de los encuestados. Valoraron positivamente su importancia en el proceso de formación, porque favorece el adiestramiento en diversas técnicas y habilidades, refuerza contenidos teóricos de la asignatura y, en definitiva, es una forma útil de aprendizaje (Escalona y Loscertales, 2005a: 39).

En las diferentes encuestas contrasta la alta valoración de la competencia trabajo en equipo con la percepción de que su enseñanza no está adecuadamente cubierta en la Universidad. Así queda reflejado en la encuesta incluida en el proyecto de título de grado en Geografía, en relación con las necesidades formativas de los perfiles "Ordenación del Territorio y desarrollo territorial", "Análisis territorial de la población y demografía" y "Estudios y análisis de ámbitos regionales" (Agencia..., op. cit: 2006). 
También en la encuesta a los estudiantes, éstos ponen de relieve deficiencias en los métodos de enseñanza utilizados. Son especialmente críticos con la ayuda que reciben al plantear los trabajos, con la valoración que merece su esfuerzo por parte de los profesores y con la forma en que se constituyen los grupos de trabajo (Escalona y Loscertales, 2005a: 40). De todo lo expuesto se deduce que la inserción de la competencia trabajo en equipo en el aprendizaje de los futuros geógrafos es oportuna y supone también un interesante reto para los profesores.

\section{Pautas para el diseño de la enseñanza y el aprendizaje de la competencia trabajo en equipo en las asignaturas del grado de Geografía}

El aprendizaje de la competencia trabajo en equipo puede realizarse en cualquiera de los niveles y bloques de contenidos de la titulación en el marco de las actividades académicas habituales -clases magistrales, seminarios, prácticas de campo y laboratorio, realización de proyectos-. Todos estos contextos favorecen que la competencia se enseñe, se practique y pueda ser evaluada. Para que el diseño del aprendizaje sea adecuado, debe realizarse previamente un diagnóstico acerca de la situación de los estudiantes. Éste ayudará a identificar cuales son sus principales carencias y, por lo tanto, permitirá conocer también cuáles son sus necesidades formativas. Considerando las reflexiones anteriores, puede servir de orientación eficaz la realización una encuesta al iniciar el curso académico entre los matriculados en la una determinada asignatura o en un conjunto de las mismas. Su estructura y complejidad dependerá de los recursos de que disponga el profesor o los profesores que van a llevar a cabo esta actividad. A modo de ejemplo, remitimos a la encuesta ya citada (GIDOCUZ, 2003; Escalona y Loscertales, 2005).

En el diseño del aprendizaje de la competencia trabajo en equipo, como para el de cualquier otra competencia, es aconsejable seguir tres etapas interrelacionadas entre sí: Identificación y secuenciación de los resultados del aprendizaje, determinación de las pruebas o evidencias en las que se basará su evaluación y selección de las actividades mediante las cuales va a facilitarse la consecución de los resultados previstos y la superación de la evaluación (Rodríguez, 2000; Cruz, 2004; Moon, 2004). Debe de procurarse que haya una adecuada y coherente relación entre los objetivos que se pretenden alcanzar, las actividades académicas diseñadas para lograrlos, y las evidencias y procedimientos elegidos para evaluar si el estudiante ha obtenido o no los resultados esperados (Wagenaar, 2003; Tuning Management Committee, 2004).

Para el desarrollo del proceso de enseñanza-aprendizaje es muy útil disponer de guías y otros materiales de apoyo a los estudiantes para que los consulten frecuente- 
mente como documentos de referencia. Para ello elaboramos una Guía para trabajar mejor en equipo (Escalona y Loscertales, 2005'), a la que haremos referencia posteriormente.

Hay que procurar que los estudiantes se involucren plenamente y que se sientan responsables de su propio proceso de aprendizaje. En este sentido, la presentación y realización de la encuesta de diagnóstico puede ser muy útil ya que les ayudará a reflexionar y tomar conciencia de sus carencias y a valorar después más cada uno de los sucesivos logros.

\subsection{Identificación de los resultados de aprendizaje}

El elevado potencial formativo de la competencia trabajo en equipo supone que sean muchas las facetas que el estudiante de Geografía debe aprender a poner en práctica para alcanzar el pleno dominio de la misma ( $v$. tabla 1).

Tabla 1. Aspectos importantes del trabajo en equipo. Elaboración propia a partir de Bradford, 2000 y Escalona y Loscertales, 2005a.

- Saber constituir un equipo de trabajo.

- Distribuir las responsabilidades de modo correcto entre los miembros del equipo.

- Cooperar de manera eficaz en la realización de un proyecto.

- Cumplir las tareas que le sean asignadas.

- Gestionar adecuadamente el tiempo disponible.

- Comunicarse con los demás miembros del equipo siempre que sea necesario.

- Ejercer funciones de dirección o liderazgo.

- Reflexionar con sinceridad sobre el funcionamiento del equipo.

- Analizar con rigor el trabajo realizado.

- Evaluarlo críticamente en equipo.

Por ello, el diseño de la enseñanza de esta competencia debe comenzar con la especificación de los resultados concretos que como profesores queremos que alcancen nuestros estudiantes. Estos resultados deben hacerse explícitos en los programas de las asignaturas de Geografía ${ }^{1}$. También es aconsejable que los resultados de aprendizaje aparezcan secuenciados por niveles. En la tabla 2 establecemos resultados para el nivel 1 (básico) y el 2 (avanzado), buscando un adecuado equilibrio entre el curso en que se encuentra el estudiante y el nivel de exigencia. Los profesores pueden adaptar los enunciados al perfil de sus estudiantes y asignaturas.

\footnotetext{
1 Los resultados pueden ser perfectamente asumidos por profesores que enseñen estas competencias genéricas en asignaturas de titulaciones afines. Este ha sido el objetivo de nuestrá participación en el I Congreso de Innovación Docente Universitaria convocado por lá Universidad de Zaragoza (v. Escalona y Loscertales, 2006).
} 
Los resultados de aprendizaje enunciados de la tabla 2 hacen referencia a aspectos relacionados con la participación de los estudiantes como miembros de un equipo, pero el dominio de esta competencia debe apreciarse también en la calidad del informe escrito, monografía, exposición oral o panel, que los estudiantes presentan. Por ese motivo, se incorpora también como resultado de aprendizaje que el estudiante contribuya a que el producto fruto del trabajo del equipo cumpla los requisitos científicos y académicos esperados.

Tabla 2. Resultados del aprendizaje para la competencia trabajo en equipo.

Elaboración propia a partir de Escalona y Loscertales, 2005a.

\begin{tabular}{|l|l|}
\hline Nivel & Resultado del aprendizaje \\
\hline 1 (básico) & $\begin{array}{l}\text { Es capaz de trabajar con compañeros a los que conoce y con los que tiene } \\
\text { relación previa. } \\
\text { Realiza las tareas que le son asignadas dentro del equipo. }\end{array}$ \\
\hline 2 (avanzado) & $\begin{array}{l}\text { Es capaz de ejercer el liderazgo o cualquier otra función de responsabili- } \\
\text { dad dentro del equipo. } \\
\text { Toma iniciativas respondiendo a otras aportaciones de sus compañeros o } \\
\text { del profesor. } \\
\text { Contribuye a que la investigación o proyecto realizado en equipo sea de la } \\
\text { calidad esperada. }\end{array}$ \\
\hline
\end{tabular}

\subsection{La evaluación del aprendizaje}

La comprobación de si los resultados de aprendizaje propuestos se han alcanzado, requiere que se solicite a los estudiantes dos tipos de pruebas o evidencias: una encaminada a valorar su rendimiento dentro del equipo y otra, sobre la calidad de lo realizado conjuntamente. Las primeras constituyen las evidencias del proceso de trabajo en equipo y las segundas, del producto fruto del mismo. Proponemos diversos ejemplos de cuáles podrían ser algunas de las pruebas o evidencias según el nivel, básico o avanzado, del estudiante (v. Tabla 3 ).

Las pruebas sugeridas para evaluar la calidad del producto fruto del trabajo en equipo, son habituales en el ámbito académico. Más compleja resulta la evaluación del proceso, es decir, de si el estudiante ha aprendido a trabajar como miembro de un equipo (v. tabla 1). En la bibliografía especializada sobre el aprendizaje de competencias en Geografía (Gibbs, 1994; Bradford, 2000) se recomienda que el equipo presente una pequeña memoria sobre el funcionamiento del grupo. Para facilitar su realización, es conveniente proporcionales algunas pautas (v. Tabla 4). 
Tabla 3. Relación de evidencias para la evaluación de los aprendizajes. Elaboración propia.

\begin{tabular}{|c|c|c|}
\hline $\begin{array}{l}\text { Nivel de } \\
\text { aprendizaje }\end{array}$ & & Evidencias para la evaluación \\
\hline \multirow{3}{*}{ Básico } & \multirow{2}{*}{$\begin{array}{l}\text { Sobre el } \\
\text { producto } \\
\text { del } \\
\text { aprendizaje }\end{array}$} & $\begin{array}{l}\text { Trabajo de carácter bibliográfico sobre una cuestión del pro- } \\
\text { grama. }\end{array}$ \\
\hline & & $\begin{array}{l}\text { Exposición oral de los resultados de una síntesis bibliográfica } \\
\text { sencilla. }\end{array}$ \\
\hline & $\begin{array}{l}\text { Sobre el } \\
\text { proceso }\end{array}$ & $\begin{array}{l}\text { Memoria descriptiva sencilla acerca del modo en que el equipo } \\
\text { ha trabajado. } \\
\text { Trabajo de carácter bibliográfico o empírico sobre una cuestión } \\
\text { más compleja que en el nivel anterior. }\end{array}$ \\
\hline \multirow{3}{*}{ Avanzado } & \multirow{2}{*}{$\begin{array}{l}\text { Sobre el } \\
\text { producto } \\
\text { del } \\
\text { aprendizaje }\end{array}$} & $\begin{array}{l}\text { Exposición oral de una investigación de carácter aplicado rela- } \\
\text { cionada con la asignatura. }\end{array}$ \\
\hline & & Panel sobre un tema cuyo estudio se ha encargado al equipo. \\
\hline & $\begin{array}{l}\text { Sobre el } \\
\text { proceso }\end{array}$ & $\begin{array}{l}\text { Memoria descriptiva completa y razonada acerca del modo en } \\
\text { que el equipo ha trabajado. }\end{array}$ \\
\hline
\end{tabular}

Tabla 4. Esquema orientativo de los contenidos de una memoria para la evaluación de la competencia trabajo en equipo.

\begin{tabular}{|c|c|}
\hline $\begin{array}{l}\text { Elementos de la } \\
\text { memoria }\end{array}$ & Contenidos \\
\hline Índice & Relación de documentos incluidos. \\
\hline \multirow{5}{*}{$\begin{array}{l}\text { Aspectos } \\
\text { contemplados }\end{array}$} & Cumplimiento de responsabilidades y tareas. \\
\hline & Distribución y gestión del tiempo de trabajo. \\
\hline & Ambiente de trabajo entre los miembros del grupo. \\
\hline & Contribución personal a la calidad del trabajo común. \\
\hline & Otras cuestiones. \\
\hline \multirow{3}{*}{$\begin{array}{l}\text { Documentos } \\
\text { complementarios }\end{array}$} & Cronograma. \\
\hline & Actas de las reuniones celebradas. \\
\hline & Autoevaluación. \\
\hline
\end{tabular}

Fuente: Elaboración propia a partir de Guía para trabajar mejor en equipo (Gidocuz, 2005). 
En la tabla 5 proporcionamos algunos criterios para la evaluación de la memoria que cada profesor puede adaptar del modo que considere más oportuno. La calificación deberá ajustarse según el peso asignado por el profesor a esta prueba en el conjunto de la asignatura.

Tabla 5. Ejemplo de criterios para evaluar las memorias sobre el funcionamiento de un equipo de trabajo formado por estudiantes.

\begin{tabular}{|l|l|l|l|l|}
\hline & 1 & 0 & 0,5 & \\
\hline Criterios de evaluación & & & & \\
\hline Adecuada estructura y presentación & & & & $\begin{array}{l}\text { Mal estructurada, mal presentada o } \\
\text { ambas. }\end{array}$ \\
\hline Reflexión completa & & & & Se omiten aspectos de interés. \\
\hline Reflexión crítica & & & & No hay sentido crítico. \\
\hline Reflexión coherente & & & & No es coherente. \\
\hline $\begin{array}{l}\text { Correcta cumplimentación de los } \\
\text { documentos complementarios }\end{array}$ & & & & Incorrecta cumplimentación. \\
\hline
\end{tabular}

Fuente: Elaboración propia a partir de Guía para trabajar mejor en equipo (Gidocuz, 2005).

\subsection{Actividades para el aprendizaje y contexto de realización}

Una vez establecidos los resultados de aprendizaje, los criterios de evaluación y las evidencias que van a ser evaluadas, habrá que diseñar las actividades que servirán para que los estudiantes de Geografía adquieran o refuercen la competencia trabajo en equipo según el nivel establecido (Moon, 2004). La tabla 6 presenta una relación de actividades encaminadas a conseguir estos objetivos. Se ha procurado que fueran variadas para que, de este modo, favorecieran la práctica de las diferentes capacidades relacionadas con el trabajo en equipo. El profesor podrá seleccionar las que le parezcan más adecuadas. Los niveles básico y avanzado los marcará mediante un diferente grado de complejidad y de exigencia (Tabla 6).

Hay que asignar a las diferentes actividades el tiempo suficiente para ser explicadas a los estudiantes y para que éstos puedan llevarlas a cabo. La primera de las actividades de la tabla -el debate- permite completar el diagnóstico y conocer tanto el interés de los estudiantes por esta competenciä como sus experiencias anteriores. Sirve también de importante ayuda para estimularles en su proceso de aprendizaje personal. Diferentes autores proporcionan algunas pautas para su realización (Gibbs, 1994; Bradford, 2000). Puede consistir en una breve sesión realizada en clase, en la que se analice la experiencia previa de los estudiantes y se les invite a tratar cuestio- 
nes como: ¿Qué es un trabajo en equipo? ¿Qué ventajas tiene esta forma de trabajar? ¿Cuáles son sus inconvenientes? ¿Son adecuados los sistemas de formación de equipos que se utilizan en clase? ¿Qué problemas plantea la organización del tiempo o el reparto de tareas? ¿Se responsabilizan por igual todos los miembros de un equipo?

La segunda de las actividades de aprendizaje que se propone consiste en la distribución y explicación en clase de diferentes materiales que ayuden a trabajar mejor en equipo. Son muy interesantes algunas publicaciones recientes sobre trabajo en equipo, y otras competencias genéricas, enfocadas a los estudiantes de Geografía (Kneale, 2003). Su consulta puede ayudar a elaborar unos materiales propios, adaptados a los resultados que pretende conseguir.

Tabla 6. Actividades para el aprendizaje de la competencia trabajo en equipo.

- Debate entre los estudiantes sobre su experiencia al trabajar en equipo con otros compañeros.

- Presentación y explicación de los materiales para el aprendizaje.

- Sesiones de trabajo en grupo supervisadas por el profesor.

- Presentación al profesor de breves informes sobre el funcionamiento del equipo y los resultados obtenidos.

Es importante que los materiales sean una herramienta práctica y eficaz que facilite el aprendizaje de la competencia y que los estudiantes los consulten con frecuencia. Por ello, sus contenidos deben ser breves y concisos y hacer referencia a los aspectos seleccionados como resultados de aprendizaje, y, en general, a las situaciones y problemas habituales a los que se enfrenta un grupo de personas que trabajan en un mismo equipo. La Guia para trabajar mejor en equipo, antes citada, se compone de ocho fichas temáticas (Tabla 7) en las que se contiene una breve explicación de los diferentes aspectos.

La guía proporciona también la ayuda necesaria para cumplimentar los diferentes documentos requeridos en la memoria para la evaluación del funcionamiento del equipo (v. Tabla 4), al incorporar diversas plantillas para el registro de las actividades realizadas, la elaboración del cronograma del trabajo y para realizar la autoevaluación (Figura 1). 
Tabla 7. Contenidos de la Guía para trabajar mejor en equipo (Gidocuz, 2005).

\begin{tabular}{l}
\hline \multicolumn{1}{|c|}{ Índice } \\
\hline 1. Presentación de la guía y consejos de utilización. \\
2. Indicaciones de utilidad para trabajar en equipo. \\
Ficha n. ${ }^{\circ}$ 1. La formación del equipo. \\
Ficha n. ${ }^{\circ}$ 2. La elección del tema. \\
Ficha $n$. $^{\circ}$ 3. Reparto de responsabilidades y tareas. \\
Ficha n. ${ }^{\circ}$ 4. La distribución y la gestión del tiempo de trabajo. \\
Ficha n. ${ }^{\circ}$ 5. El funcionamiento y la comunicación interna del equipo. \\
Ficha nn. ${ }^{\circ}$ 6. Sugerencias para trabajar mejor en equipo. \\
Ficha n. ${ }^{\circ}$ 7. Propuestas para reflexionar sobre el funcionamiento del equipo. \\
Ficha n. ${ }^{\circ}$ 8. Evaluación conjunta del funcionamiento del equipo. \\
3. Pautas para la elaboración de la memoria final. \\
4. Criterios de valoración de la memoria final.
\end{tabular}

Para que la guía sea realmente útil, es importante que en la sesión de presentación queden claros los diferentes aspectos de su contenido, prestando una especial atención a las fases en las que dicha actividad se va a desarrollar. En la tabla 8 se exponen los principales aspectos del desarrollo de esta sesión.

La realización de tutorías o sesiones para supervisar el aprendizaje es una actividad fundamental. Por ello deben ser obligatorias y convocadas con la suficiente antelación para que los estudiantes puedan prepararlas suficientemente dando cuenta en

Tabla 8. Desarrollo de la actividad centrada en el reparto y comentario de materiales para el aprendizaje.

1. Entrega del material a cada uno de los estudiantes.

2. Breve explicación por el profesor de su finalidad y de los aspectos básicos recogidos en la guía.

3. Lectura individual de todo o de parte del material.

4. Aclaración a los estudiantes de diferentes aspectos requeridos por ellos acerca del material distribuido.

5. Explicación de las plantillas de los documentos que deberán ir elaborando y adjuntar al final como prueba de su aprendizaje.

6. Reflexión sobre la importancia de una correcta gestión del tiempo.

7. Explicación del procedimiento que se seguirá en las tutorías periódicas de seguimiento, incluida su preparación.

8. Conclusiones de la sesión. 


\section{Documento $\mathrm{n}^{\circ}$ 4. Evaluación del equipo}

Puntúa de 0 a 10 los siguientes aspectos de vuestro trabajo:

1) La idoneidad del sistema de formacion del cquipo

$\square 0 \square 1 \quad \square 2 \quad \square 3 \quad \square 4 \quad \square 5 \quad \square 6 \quad \square 7 \quad \square 8 \quad \square 9 \quad \square 10$

2) La superación de las dificultades surgidas en la colaboración con personas con las que no se trabaja habitualmente
Q $\mathrm{Q} 1$
$\square 2$
$\square 3 \square 4 \quad \square 5$
$\square 6$
778
$\square 9 \square 10$

3) El grado de cumplimientos de las responsabilidades asignadas

$\square 0, \square 1 \square 2 \quad \square 3 \quad \square 4 \quad \square 5 \quad \square 6 \quad \square 7 \quad \square 8 \quad \square 9 \quad \square 10$

4) El grado de cumplimiento de las tareas asignadas

$\square 0 \square 1 \quad \square 2 \quad \square 3 \square 4 \quad \square 5 \square \square \quad \square 7 \quad \square 8 \quad \square 9, \square 10$

5) El grado de cumplimiento de los plazos

$\square 0 \square 1, \square 2, \square 3, \square 4 . \square 5, \square 6, \square 7, \square 8 \square \square 9 \square 10$

6) La idoneidad del número de reuniones

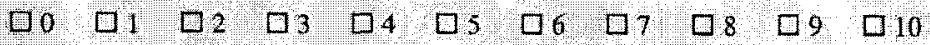

7) La eficacia de las reuniones mantenidas

$\square 0, \square 1 \quad \square 2 \quad \square 3 \quad \square 4 \square 5 \quad \square 6 \quad \square 7 \quad \square 8 \quad \square 9 \quad \square 10$

8) Ia transmision de ideas y conocimientos dentro del equipo

$\square 0, \square 1, \square 2, \square 3 \quad \square 4 \quad \square s \quad \square 6 / \square 7 \quad \square 8 \quad \square 9 \square \square 10$

9) El talante negocindor de los miembros del equipo

$\square 0 \square \square \square 2 \square 3 \quad \square 4 \square 5 \quad \square 6, \square 7 \quad \square 8 \square 9: \square 10$

$10)$ El ambiente de trabajo dentro del equipo
$\mathrm{\square} 0$
$\square 142$
प 3
$\square 4 \square 5 \square 6$
$\square 7 \square 8$
$\square 9 \quad \square 10$

Nombre y firma de los miembros del equipo

Figura 1. Formulario para la autoevaluación del equipo (Gidouz, 2005). 
el informe elaborado para la misma de los diferentes aspectos de su trabajo. Estos informes son muy eficaces ya que favorecen una interesante reflexión crítica previa por parte de los estudiantes.

\section{Conclusión}

El punto de partida de este trabajo ha sido la próxima entrada en vigor de la convergencia universitaria europea y el establecimiento efectivo del Espacio Europeo de Educación Superior. El aprendizaje de competencias específicas y genéricas va a ser un referente común para la formación de los estudiantes de las diferentes titulaciones y sistemas universitarios europeos, y "si bien éstas no son las únicas consideraciones para el desarrollo del programa de estudio y titulaciones, son de vital importancia" (VV.AA., 2003: 81). Es lógico que esta cuestión suscite controversia entre los profesionales de la enseñanza universitaria, pero enseñar debe consistir no sólo en ir transmitiendo conocimientos teórico - prácticos cada vez más complejos, sino en formar en el más amplio sentido del término a los ciudadanos del futuro.

Ciertamente, habrá que estructurar los contenidos en la nueva titulación de Geografía, como en otras titulaciones, de modo que puedan dar cabida a los aprendizajes de las competencias genéricas y específicas seleccionadas. A las autoridades académicas universitarias responsables del diseño de la titulación, corresponderá dar previamente las pautas oportunas sobre la estructura de las enseñanzas; es decir, sobre su organización en cursos, módulos y créditos. El reto que se planteará entonces a los profesores será justamente, seleccionar y aplicar las metodologías docentes más adecuadas para que las competencias, genéricas y específicas, de los titulados sean las que la sociedad necesita. Para facilitar esta tarea, en este artículo se presentan diversas pautas centradas en el aprendizaje de una competencia genérica esencial para los estudiantes de Geografía, como es el trabajo en equipo. En las páginas precedentes se han incorporado diversas indicaciones sobre cómo y en qué contextos los profesores interesados pueden enseñar a sus estudiantes esta competencia; sobre cómo favorecer su práctica. Igualmente se ha tratado de proporcionar variados procedimientos y sugerencias para la evaluación. Tales pautas son fácilmente trasladables, con pequeños ajustes, a la enseñanza y aprendizaje de otras competencias genéricas.

Trabajar de este modo no es fácil, ya que existen múltiples inconvenientes reales para trasladar a las aulas lo que en principio podrían considerarse meras quimeras, reflexiones más o menos utópicas. Pero en nuestro modo de actuar como profesores no podemos olvidar la importancia que tiene mejorar día a día los métodos de ense- 
ñanza. En este sentido debe reconocerse que el proceso de convergencia nos ha brindado a todos la ocasión de reflexionar sobre la calidad de nuestro trabajo y de asumir nuevos e ilusionantes retos.

\section{Bibliografía}

Agencia Nacional de Evaluación de la Calidad y de Acreditación (2004): Título de grado en Geografia y Ordenación del Territorio. Madrid.

Bradford, M. (2000): Improving studentsteam and personal skills. Gloucester, Geography Discipline Network, Geography \& Environmental Research Unit, and Cheltenham \& Gloucester College of Higher Education.

Comisión para la renovación de las metodologías educativas en la Universidad (2006): Propuestas para la renovación de las metodologias educativas en la Universidad. Madrid, Ministerio de Educación, Secretaría General de Universidades.

Cruz Tomé, M.A. de la (2004): El proceso de aprendizaje-enseñanza de competencias. Zaragoza, Universidad de Zaragoza, Instituto de Ciencias de la Educación.

Escalona Orcao, A.I. y Loscertales Palomar, B. (2005a): Actividades para la enseñanza y el aprendizaje de competencias en el marco del Espacio Europeo de Educación Superior. Zaragoza, Prensas Universitarias de Zaragoza, col. Textos docentes, $n^{\circ} 110$.

Escalona Orcao, A.I. y Loscertales Palomar, B. (2005b): Los aprendizajes de competencias genéricas de los futuros graduados en Geografía: Una reflexión inicial y pautas para su diseño y enseñanza. Geographicalia, nueva época, 48 (en prensa).

Escalona Orcao, A.I. y Loscertales Palomar, B. (2006): Buenas prácticas en la enseñanza y el aprendizaje de competencias genéricas. Zaragoza, Universidad de Zaragoza, I Jornadas de Innovación Docente, Tecnologías de la Información y la Comunicación e Investigación Educativa de la Universidad de Zaragoza (en prensa).

Gibbs, G. (1994): Learning in teams. A student manual. Oxford, Oxford Centre for Staff development.

GIDOCUZ (Escalona, A; Loscertales, B; Lardiés, R. y Lacosta, A.) (2003): "Aportación metodológica para favorecer el aprendizaje de competencias genéricas en la licenciatura de Geografía". En Marrón Gaite, Ma.J., Moraleda Nieto, C. y Rodríguez de Gracia, H. (eds.): La enseñanza de la Geografia ante las nuevas demandas sociales. Toledo, Grupo de Didáctica de la Asociación de Geógrafos Españoles, Universidad de Castilla-La Mancha y Escuela Universitaria de Magisterio de Toledo, 419-432.

GIDOCUZ (Escalona, A; Loscertales, B; Lardiés, R. y Lacosta, A.) (2005): Guía para trabajar mejor en equipo. En Escalona Orcao, A.I. y Loscertales palomar, B. (2005a): Actividades para la enseñanza y el aprendizaje de competencias en el marco del Espacio Europeo de Educación Superior. Zaragoza, Prensas Universitarias de Zaragoza, col. Textos docentes, $n^{\circ} 110$, pp. 89 a $\mathbf{1 0 6 .}$

Gravestock, P. y Healey, M. (2000): "Editors preface". En Burkill, S., Corey, D. y Healey, M.: Improving students ${ }^{-}$commu- 
nication skills. Gloucester, Geography Discipline Network, Geography \& Environmental Research Unit, and Cheltenham \& Gloucester College of Higher Education, vii-x.

Kneale, P. (2003): Study skills for Geograpby students. A practical guide. Arnold.

Lasnier, F. (2000): Réussir la formation per competences. Montréal, Guerin.

Moon, L. (2004): Linking levels, Learning Outcomes and Assesment Criteria. Edimburgo, Seminario Tuning sobre resultados de aprendizaje.

Moreno Jiménez, A. y Marrón Gaite, M.J. (1995): Enseñar Geografía. De la teoria a la práctica. Madrid, Síntesis.

Rodríguez Espinar, S. (2000): El diseño de proyectos de innovación docente. Zaragoza, Instituto de Ciencias de la Educación.

Tarroja, A. (2005): Los perfiles profesionales de los geógrafos y el futuro de las ense- ñanzas universitarias de Geografía. Boletin Colegio de Geógrafos, 5, 1-2.

Tuning Management Committee (2004): Student workload, teaching methods and learning outcomes: the tuning approach. En Proyecto Tuning, fase 2 www.relint. deusto.es/TUNINGProject/index_english. htm (consulta: 20/7/2005s).

Wagenaar, R. (2003): The tuning methodology. Learning outcomes and competences. (www.relint.deusto.es/TUNINGProject/pr esentations/presentations_phase2/PRESENTACION3.pdf) (consulta: 10/9/2005)

V.AA (2001): Marco Pedagogico UD. Universidad de Deusto, Bilbao (http://iajbs.org/ Images/AEImages/Marco_Pedagogico.pdf) (consulta: 13/10/2005).

VV.AA. (2003): Tuning educational structures in Europe. Informe Final, Proyecto Piloto fase 1 (www.relint.deusto.es) 